\title{
Sensitivity of the DARWIN observatory to the neutrinoless double beta decay of ${ }^{136} \mathrm{Xe}$
}

\section{DARWIN Collaboration ${ }^{\mathrm{c}}$}

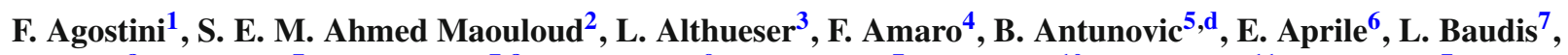

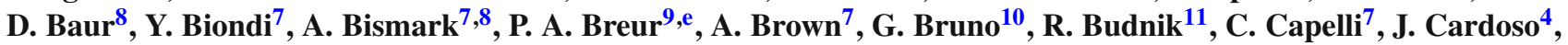
D. Cichon ${ }^{12}$, M. Clark ${ }^{13}$, A. P. Colijnn, f, J. J. Cuenca-García ${ }^{14}$, J. P. Cussonneau ${ }^{15}$, M. P. Decowski ${ }^{9}$, A. Depoian ${ }^{13}$, J. Dierle ${ }^{8}$, P. Di Gangi ${ }^{1}$, A. Di Giovanni ${ }^{10}$, S. Diglio ${ }^{15}$, J. M. F. dos Santos ${ }^{4}$, G. Drexlin ${ }^{16}$, K. Eitel $^{14}$, R. Engel $^{14}$, A. D. Ferella ${ }^{17,18}$, H. Fischer ${ }^{8}$, M. Galloway ${ }^{7}$, F. Gao ${ }^{6}$, F. Girard ${ }^{7}$, F. Glück ${ }^{14}$, L. Grandi ${ }^{19}$, R. Größle ${ }^{14}$, R. Gumbsheimer ${ }^{14}$, S. Hansmann-Menzemer 20 , F. Jörg ${ }^{12}$, G. Khundzakishvili' ${ }^{13}$, A. Kopec ${ }^{13}$, F. Kuger ${ }^{8, a}$, L. M. Krauss ${ }^{21}$, H. Landsman ${ }^{11}$, R. F. Lang ${ }^{13}$, S. Lindemann ${ }^{8}$, M. Lindner ${ }^{12}$, J. A. M. Lopes ${ }^{4, g}$, A. Loya Villalpando ${ }^{9}$, C. Macolino ${ }^{22}$, A. Manfredini ${ }^{7}$, T. Marrodán Undagoitia ${ }^{12}$, J. Masbou ${ }^{15}$, E. Masson $^{22}$, P. Meinhardt ${ }^{8}$, S. Milutinovic ${ }^{5}$, A. Molinario ${ }^{23}$, C. M. B. Monteiro ${ }^{4}$, M. Murra ${ }^{3}$, U. G. Oberlack ${ }^{24}$, M. Pandurovic ${ }^{5}$, R. Peres ${ }^{7}$, J. Pienaar ${ }^{19}$, M. Pierre ${ }^{15}$, V. Pizzella ${ }^{12}$, J. Qin ${ }^{13}$, D. Ramírez García ${ }^{8}$, S. Reichard $^{7}$, N. Rupp ${ }^{12}$, P. Sanchez-Lucas ${ }^{7, b}$, G. Sartorelli ${ }^{1}$, D. Schulte ${ }^{3}$, M. Schumann ${ }^{8}$, L. Scotto Lavina ${ }^{2}$, M. Selvi ${ }^{1}$, M. Silva ${ }^{4}$, H. Simgen ${ }^{12}$, M. Steidl ${ }^{14}$, A. Terliuk ${ }^{20}$, C. Therreau ${ }^{15}$, D. Thers ${ }^{15}$, K. Thieme ${ }^{7}$, R. Trotta ${ }^{25, h}$, C. D. Tunnell ${ }^{26}$, K. Valerius ${ }^{14}$, G. Volta ${ }^{7}$, D. Vorkapic ${ }^{5}$, C. Weinheimer ${ }^{3}$, C. Wittweg ${ }^{3}$, J. Wolf ${ }^{16}$, J. P. Zopounidis ${ }^{2}$, K. Zuber ${ }^{27}$

${ }^{1}$ Department of Physics and Astronomy, University of Bologna and INFN-Bologna, 40126 Bologna, Italy

${ }^{2}$ LPNHE, Université Pierre et Marie Curie, Université Paris Diderot, CNRS/IN2P3, 75252 Paris, France

${ }^{3}$ Institut für Kernphysik, Westfälische Wilhelms-Universität Münster, 48149 Münster, Germany

${ }^{4}$ LIBPhys, Department of Physics, University of Coimbra, 3004-516 Coimbra, Portugal

5 Vinca Institute of Nuclear Science, University of Belgrade, Mihajla Petrovica Alasa 12-14., Belgrade, Serbia

${ }^{6}$ Physics Department, Columbia University, New York, NY 10027, USA

${ }^{7}$ Physik-Institut, University of Zurich, 8057 Zurich, Switzerland

${ }^{8}$ Physikalisches Institut, Universität Freiburg, 79104 Freiburg, Germany

${ }^{9}$ Nikhef and the University of Amsterdam, Science Park, 1098XG Amsterdam, The Netherlands

${ }^{10}$ New York University Abu Dhabi, Abu Dhabi, United Arab Emirates

${ }_{11}$ Department of Particle Physics and Astrophysics, Weizmann Institute of Science, 7610001 Rehovot, Israel

12 Max-Planck-Institut für Kernphysik, 69117 Heidelberg, Germany

13 Department of Physics and Astronomy, Purdue University, West Lafayette, IN 47907, USA

${ }^{14}$ Institute for Nuclear Physics (IKP), Karlsruhe Institute of Technology (KIT), 76344 Eggenstein-Leopoldshafen, Germany

15 SUBATECH, IMT Atlantique, CNRS/IN2P3, Université de Nantes, 44307 Nantes, France

${ }^{16}$ Institute of Experimental Particle Physics (ETP), Karlsruhe Institute of Technology (KIT), 76344 Eggenstein-Leopoldshafen, Germany

17 Department of Physics and Chemistry, University of L'Aquila, 67100 L'Aquila, Italy

18 INFN-Laboratori Nazionali del Gran Sasso and Gran Sasso Science Institute, 67100 L'Aquila, Italy

19 Department of Physics \& Kavli Institute for Cosmological Physics, University of Chicago, Chicago, IL 60637, USA

${ }^{20}$ Physikalisches Institut, Ruprecht-Karls-Universität Heidelberg, Heidelberg, Germany

21 The Origins Project Foundation, Phoenix, AZ 85020, USA

${ }^{22}$ Université Paris-Saclay, CNRS/IN2P3, IJCLab, 91405 Orsay, France

23 INFN-Laboratori Nazionali del Gran Sasso and Gran Sasso Science Institute, 67100 L'Aquila, Italy

${ }^{24}$ Institut für Physik \& Exzellenzcluster PRISMA+, Johannes Gutenberg-Universität Mainz, 55099 Mainz, Germany

${ }^{25}$ Department of Physics, Imperial Centre for Inference and Cosmology, Imperial College London, London SW7 2AZ, UK

26 Department of Physics and Astronomy, Rice University, Houston, TX 77005, USA

${ }^{27}$ Institute for Nuclear and Particle Physics, TU Dresden, 01069 Dresden, Germany 
Abstract The DARWIN observatory is a proposed nextgeneration experiment to search for particle dark matter and for the neutrinoless double beta decay of ${ }^{136} \mathrm{Xe}$. Out of its $50 \mathrm{t}$ total natural xenon inventory, $40 \mathrm{t}$ will be the active target of a time projection chamber which thus contains about $3.6 \mathrm{t}$ of ${ }^{136} \mathrm{Xe}$. Here, we show that its projected half-life sensitivity is $2.4 \times 10^{27}$ years, using a fiducial volume of $5 \mathrm{t}$ of natural xenon and 10 years of operation with a background rate of less than 0.2 events/( $\mathrm{t}$ - year) in the energy region of interest. This sensitivity is based on a detailed Monte Carlo simulation study of the background and event topologies in the large, homogeneous target. DARWIN will be comparable in its science reach to dedicated double beta decay experiments using xenon enriched in ${ }^{136} \mathrm{Xe}$.

\section{Introduction}

Neutrinos are the only known elementary particles that are Majorana fermion candidates, implying that they would be their own antiparticles. The most sensitive probe for the Majorana nature of neutrinos is an extremely rare nuclear decay process called neutrinoless double beta decay $(0 \nu \beta \beta)$, where a nucleus with mass number $\mathrm{A}$ and charge $\mathrm{Z}$ decays by emitting only two electrons and changes its charge by two units $(\mathrm{A}, \mathrm{Z}) \longrightarrow(\mathrm{A}, \mathrm{Z}+2)+2 \mathrm{e}^{-}$. The observation of this decay would mean that lepton number is violated by two units and, in the standard light Majorana neutrino exchange scenario, would yield information about the neutrino mass scale via the effective neutrino Majorana mass $\left\langle m_{\beta \beta}\right\rangle=\left|\Sigma_{i} U_{e i}^{2} m_{i}\right|$. The sum is over the neutrino mass eigenstates, $m_{i}$, and $U_{e i}$, the corresponding entries in the lepton mixing matrix, which are complex numbers. The two-neutrino double beta decay mode $(2 v \beta \beta)$ is allowed in the Standard Model and has been observed in more than 10 nuclei [1]. In this case, the summed energy of the two electrons is a continuum, while for the $0 \nu \beta \beta$-decay the distinct signature is a peak at the Q-value, the mass difference between the mother and daughter nuclei.

Experiments can observe a certain decay rate in a detector. The corresponding half-life is inversely proportional to $\left\langle m_{\beta \beta}\right\rangle^{2}$,

$\frac{1}{T_{1 / 2}^{0 v}}=\frac{\left\langle m_{\beta \beta}\right\rangle^{2}}{m_{e}^{2}} G^{0 v}\left|M^{0 v}\right|^{2}$,

\footnotetext{
a e-mail: Fabian.Kuger@physik.uni-freiburg.de

b e-mail: patricia.sanchez@physik.uzh.ch

c e-mail: darwin@lngs.infn.it

d Also at University of Banja Luka, Bosnia and Herzegovina

e Now at SLAC, Menlo Park, CA 94025, USA

${ }^{\mathrm{f}}$ Also at Institute for Subatomic Physics, Utrecht University, Utrecht, The Netherlands

g Also at Coimbra Polytechnic-ISEC, Coimbra, Portugal

${ }^{\mathrm{h}}$ Also at SISSA, Data Science Excellence Department, Trieste, Italy
}

assuming that the decay is mediated by the exchange of a light Majorana neutrino. $m_{e}$ is the mass of the electron, $G^{0 v}$ is the phase space factor, and $M^{0 v}$ is the nuclear matrix element. Recent experimental limits on $T_{1 / 2}^{0 v}$ and $\left\langle m_{\beta \beta}\right\rangle$ are of the order $T_{1 / 2}^{0 v} \geq\left(10^{25}-10^{26}\right)$ years and $\left\langle m_{\beta \beta}\right\rangle \leq(0.06-0.17) \mathrm{eV}$, using a variety of nuclei and detector technologies $[2,3]$.

A particularly suitable isotope to search for the $0 v \beta \beta$ decay with is ${ }^{136} \mathrm{Xe}$, with $Q_{\beta \beta}=(2457.83 \pm 0.37) \mathrm{keV}$ [4]. Current experiments use liquid xenon either in its pure form, EXO-200 [5], or xenon dissolved in liquid scintillator, KamLAND-Zen [6], and provide competitive constraints on the half-life. Future detectors that use xenon gas operated at high pressure, NEXT [7,8] and PandaX-III [9], will add tracking capabilities for improved background rejection, while $\mathrm{nEXO}$ [10] proposes to operate a total of $5 \mathrm{t}$ of isotopically enriched liquid xenon.

DARWIN [11] is a proposed observatory using $40 \mathrm{t}$ of liquid natural xenon (LXe) in a time projection chamber (TPC) with the primary goal of searching for particle dark matter. Here, we demonstrate that DARWIN has a similar reach to dedicated future neutrinoless double beta decay experiments. This is due to its large, homogeneous target, and its ultra-low background, coupled to the capability of the TPC to simultaneously measure the location, energy, particle type and multiplicity of an event [12].

The paper is organized as follows: in Sect. 2 we provide a brief review of the baseline design of the DARWIN detector and describe the detector model utilized in our simulation study. Section 3 addresses the signal topology and how it is used to reject background events. In Sect. 4 we discuss the expected background sources, while the resulting background spectra and rates are presented in Sect. 5. We discuss DARWIN's sensitivity to $0 \nu \beta \beta$-decay in Sect. 6 and give a summary and an outlook in Sect. 7.

\section{The DARWIN observatory}

DARWIN is a next-generation dark matter experiment that will operate a $40 \mathrm{t}$ active (50t total) liquid xenon TPC with the main goal to probe the entire experimentally accessible parameter space for weakly interacting massive particles (WIMPs) as dark matter candidates. Other physics goals include the search for the $0 \nu \beta \beta$-decay, the real-time detection of solar $p p$ neutrinos via electron scattering, the observation of supernova and solar ${ }^{8} \mathrm{~B}$ neutrinos via coherent neutrino nucleus scattering and the search for solar axions, galactic axion-like particles and dark photons.

The DARWIN detector is described in detail in [11]. In the baseline scenario, the detector is a cylindrical, two-phase (liquid and gas) xenon TPC with $2.6 \mathrm{~m}$ diameter and $2.6 \mathrm{~m}$ height. The TPC will be placed in a low-background, double- 


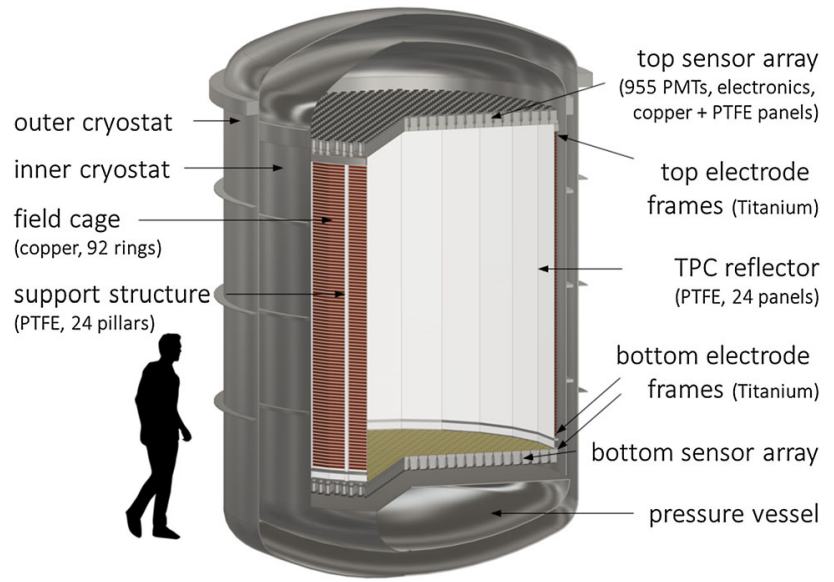

Fig. 1 Drawing of DARWIN's double-walled cryostat and TPC, showing all components considered in the simulation

walled cryostat surrounded by an instrumented water tank to shield it from the environmental radioactivity and to record the passage of cosmic muons and their secondaries as well as for neutron thermalization.

Interactions in the TPC will give rise to a prompt signal $(S 1)$ from photons and a delayed, proportional scintillation signal $(S 2)$ from electrons transported by a homogeneous drift field and extracted into the gas phase. Both signals will be detected by photosensor arrays (made of photomultiplier tubes (PMTs), silicon photomultiplier (SiPM), or new types of sensors), providing the $x-y$-z-coordinates of an interaction, as well as its energy with $<1 \% 1 \sigma$ resolution for $\mathrm{MeV}$ energy depositions. Interactions separated by more than $15 \mathrm{~mm}$ are assumed to be individually identified in event reconstruction. This allows for separation between single scatters (as expected from $0 v \beta \beta$-decays and dark matter particle interactions) and multiple scatters (as expected from many sources of backgrounds), as well as the definition of an inner (fiducial) volume with reduced background levels. The high density of the liquid xenon $\left(\sim 3 \mathrm{~g} / \mathrm{cm}^{3}\right)$ ensures a short attenuation length for $\gamma$-rays.

The final location of the DARWIN experiment is yet to be decided. A good candidate is the Gran Sasso Underground Laboratory (LNGS) in Italy. We will use its overburden in this study.

\subsection{Monte Carlo model of the detector}

For the Monte Carlo event generation and particle propagation in GEANT4 we use a realistic model of the DARWIN detector. Its details are described in the following.

The TPC is enclosed within the outer and inner titanium cryostat (shown in Fig. 1), including torispherical domes, flanges and stiffening rings to minimize the amount of material. A dome-shaped pressurizable titanium vessel is placed
Table 1 List of detector components included in the GEANT4 geometry model of DARWIN stating their material composition and total mass

\begin{tabular}{lll}
\hline Component & Material & Mass \\
\hline Outer cryostat & Titanium & $3.0 \mathrm{t}$ \\
Inner cryostat & Titanium & $2.1 \mathrm{t}$ \\
Bottom pressure vessel & Titanium & $0.4 \mathrm{t}$ \\
LXe instrumented target & LXe & $39.3 \mathrm{t}$ \\
LXe buffer outside the TPC & LXe & $9.0 \mathrm{t}$ \\
LXe around pressure vessel & LXe & $270 \mathrm{~kg}$ \\
GXe in top dome + TPC top & GXe & $30 \mathrm{~kg}$ \\
TPC reflector (3mm thickness) & PTFE & $146 \mathrm{~kg}$ \\
Structural support pillars (24 units) & PTFE & $84 \mathrm{~kg}$ \\
Electrode frames & Titanium & $120 \mathrm{~kg}$ \\
Field shaping rings (92 units) & Copper & $680 \mathrm{~kg}$ \\
Photosensor arrays (2 disks): & & \\
Disk structural support & Copper & $520 \mathrm{~kg}$ \\
Reflector + sliding panels & PTFE & $70 \mathrm{~kg}$ \\
Photosensors: 3" PMTs (1910 units) & Composite & $363 \mathrm{~kg}$ \\
Sensor electronics (1910 units) & Composite & $5.7 \mathrm{~kg}$ \\
\hline
\end{tabular}

on the inner cryostat floor to reduce the volume to be filled with liquid xenon while keeping the material budget low. A study based on previously-measured specific activities of cryostat materials $[13,14]$ showed that a cryostat made of titanium yields a lower background rate than a stainless steel cryostat of equal mechanical properties.

The inner cryostat contains the liquid xenon volume and the TPC. The TPC walls are formed by PTFE reflectors of $3 \mathrm{~mm}$ thickness with high reflectivity for the vacuum ultraviolet (VUV) scintillation light, surrounded by 92 cylindrical copper field shaping rings. The structure is reinforced with 24 PTFE support pillars. Titanium frames at the bottom and top of the TPC support the electrodes to establish drift and extraction fields. Two photosensor arrays are located at the top and bottom of the TPC cylinder, consisting of a structural copper support, a PTFE reflector disk, the VUV-sensitive photosensors and the sensors' cold electronics. Because the final sensor type is yet to be chosen for DARWIN and R\&D on light sensor options [15-18] is ongoing, the top and bottom sensors have, for the majority of simulations, been simplified to two disks which properly account for the material budget and the associated activities of radioactive isotopes. This allows for a direct comparison between a baseline scenario with PMTs and an alternative based on SiPMs.

All the major components included in the simulations are listed in Table 1. The assumed radioactivity levels of the materials are discussed in Sect. 4 and listed in Table 2. 


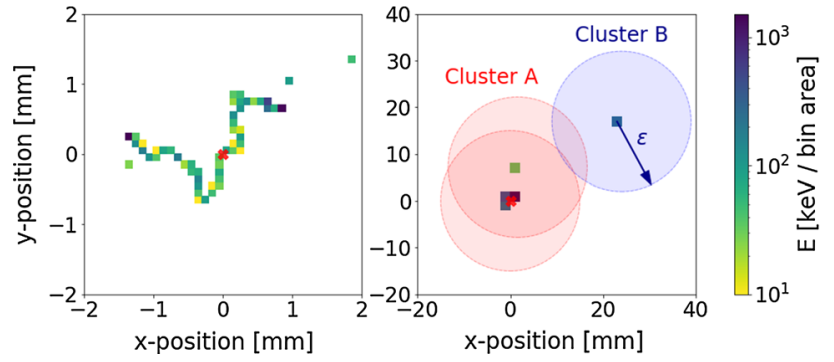

Fig. 2 Simulated energy deposition (color scale) of two different $0 \nu \beta \beta$-events in the $x-y$-plane. Left: The two electrons thermalize along two non-resolvable back-to-back tracks. The emitted Bremsstrahlung photons yield detached energy depositions. Right: A $\mathcal{O}(400 \mathrm{keV})$ photon Compton scatters $8 \mathrm{~mm}$ from the position of the decaying ${ }^{136} \mathrm{Xe}$ nucleus and travels more than $2 \mathrm{~cm}$ without energy loss before absorption. The circles indicate the boundaries of individually resolvable clusters assuming a separation threshold $\epsilon=15 \mathrm{~mm}$

\section{$30 v \beta \beta$ signal events in liquid xenon}

In a $0 v \beta \beta$-decay, the energy $Q_{\beta \beta}$ is released mainly in the form of kinetic energy of the two electrons. In liquid xenon, the electrons thermalize within $\mathcal{O}(\mathrm{mm})$ resulting in a single site (SS) signal topology, as shown in Fig. 2 (left). Bremsstrahlung photons emitted during electron thermalization travel some distance without energy deposition before scattering or being absorbed. Abundantly emitted low energy photons are likely to deposit their energy close to the decay position and remain unresolved in the DARWIN detector. Photons with energies above $300 \mathrm{keV}$ have a mean free path of more than $15 \mathrm{~mm}$ and might travel larger distances before interacting. This can result in an energy deposition which is spatially separable and can cause a false identification as a multi site (MS) event, Fig. 2 (right).

Energy depositions are therefore spatially grouped using a density-based spatial clustering algorithm [19]. An energy deposition is considered as a new cluster if its distance to any previous energy deposition is larger than our selected separation threshold $\epsilon$. Figure 3 shows the efficiency for signal acceptance and background rejection for photons and electrons with an energy of $Q_{\beta \beta}$ as a function of $\epsilon$.

The distribution of energy per electron and the angle between the two depend on the yet unknown decay mechanism. We assume a mass mixing mechanism and the most probable decay where the electrons are emitted back-to-back, each with a kinetic energy of $Q_{\beta \beta} / 2$. This assumption is compared in Fig. 3 to the predicted energy and angular distributions in the mass mixing (MM) model and a right-handed current (RHC) model presented in [20].

We assume that a spatial separation between energy depositions of $\epsilon=15 \mathrm{~mm}$ can be resolved in the DARWIN TPC. This results in a signal acceptance of $90.4 \%$ (MM: $88.7 \%$, RHC: $86.6 \%$ ) as SS events. Background events from electrons and photons with $Q_{\beta \beta}$ energy are rejected as MS with an

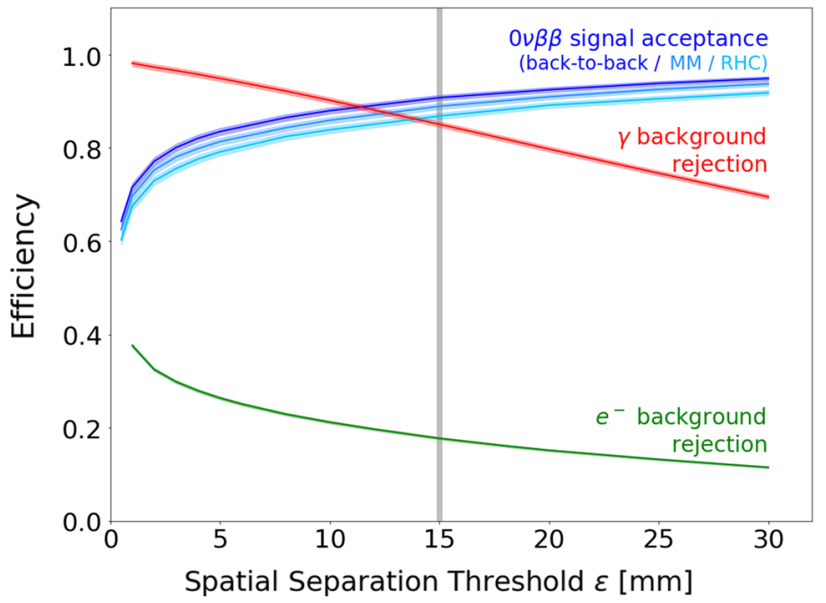

Fig. 3 Efficiency of $0 \nu \beta \beta$ signal acceptance and background rejection as a function of the spatial separation threshold $\epsilon$. The three signal lines (blue) compare different energy and angular distributions based on a back-to-back electron emission, a mass mixing (MM) mechanism and a right-handed current (RHC) model. The background rejection efficiency is shown for $\gamma \mathrm{s}$ (red) and electrons (green) with $E=Q_{\beta \beta}$. The vertical line (grey) corresponds to the value of $\epsilon$ assumed in this study. Bands indicate $\pm 2 \sigma$ uncertainties

efficiency of $17.7 \%$ and $85.1 \%$, respectively. A smaller separation threshold $\epsilon$ results in a larger fraction of misidentified $0 \nu \beta \beta$-decays. Simultaneously, electrons from $\beta$ decays and $\gamma$-ray events are more efficiently identified as MS. As we will discuss in Sect. 7, a lower spatial threshold can increase the sensitivity to $0 v \beta \beta$-decays. The decrease in signal acceptance is overcompensated by the improved background rejection.

\section{Background events in the $0 v \beta \beta$ energy range}

We discuss all background sources which contribute events within the energy range of [2.3-2.7] $\mathrm{MeV}$ around $Q_{\beta \beta}$. We consider intrinsic background events from radioactive decays (radiogenic) and those induced by cosmic neutrinos, muons and their secondaries (cosmogenic). Intrinsic events are homogeneously distributed in the liquid xenon. Likewise we study radiogenic background radiation from external sources emanating into the target.

\subsection{Homogeneously distributed intrinsic background}

The intrinsic background sources originate from noble gas isotopes or from interactions of cosmogenic particles with the xenon target:

$-{ }^{8} \mathrm{~B}$ solar neutrinos are an irreducible background source. The expected rate of $v-e^{-}$scatterings is derived assuming $\mathrm{a}^{8} \mathrm{~B}-\mathrm{v}$ flux of $\phi=(5.46 \pm 0.66) \times 10^{6} \mathrm{~cm}^{-2} \mathrm{~s}^{-1}$ [21]. The calculation of scattering cross sections follows [22]. The 
Table 2 Assumed activity levels for the simulated materials and isotopes

\begin{tabular}{lllllllll}
\hline Material & Unit & ${ }^{238} \mathrm{U}$ & ${ }^{226} \mathrm{Ra}$ & ${ }^{232} \mathrm{Th}$ & ${ }^{228} \mathrm{Th}$ & ${ }^{60} \mathrm{Co}$ & ${ }^{44} \mathrm{Ti}$ & Reference \\
\hline Titanium & $\mathrm{mBq} / \mathrm{kg}$ & $<1.6$ & $<0.09$ & 0.28 & 0.25 & $<0.02$ & $<1.16$ & {$[13]$} \\
PTFE & $\mathrm{mBq} / \mathrm{kg}$ & $<1.2$ & 0.07 & $<0.07$ & 0.06 & 0.027 & - & {$[14]$} \\
Copper & $\mathrm{mBq} / \mathrm{kg}$ & $<1.0$ & $<0.035$ & $<0.033$ & $<0.026$ & $<0.019$ & - & {$[14]$} \\
PMT & $\mathrm{mBq} / \mathrm{unit}$ & 8.0 & 0.6 & 0.7 & 0.6 & 0.84 & - & {$[14]$} \\
Electronics & mBq/unit & 1.10 & 0.34 & 0.16 & 0.16 & $<0.008$ & - & {$[14]$} \\
\hline
\end{tabular}

electron neutrino survival probability is conservatively estimated to be $P_{e e}=0.50_{-30 \%}^{+5 \%}$ for neutrinos with $E_{v}>$ $Q_{\beta \beta}$.

- ${ }^{137} \mathrm{Xe}$ from cosmogenic activation: muon-induced neutrons produced in the liquid xenon can thermalize and be captured on a ${ }^{136} \mathrm{Xe}$ nucleus, producing ${ }^{137} \mathrm{Xe}$, as measured by EXO-200 [23]. This isotope decays via a $\beta^{-}$ process with $\mathrm{Q}_{\beta}=4.17 \mathrm{MeV}$ and a half-life of $3.82 \mathrm{~min}$. Assuming the depth of LNGS and previous simulations of the muon-induced neutron flux underground [24], we estimate the muon-induced ${ }^{137} \mathrm{Xe}$ production rate in DARWIN to be $(6.9 \pm 0.4)$ atoms/(t.year). Neutrons produced in the solid materials contribute about $5 \%$ of this rate. Activation of ${ }^{136} \mathrm{Xe}$ due to radiogenic neutrons from the TPC materials has been found to be subdominant by more than two orders of magnitude. Activation of xenon in the non-shielded environment of the purification loop is non-negligible, but can be efficiently suppressed by a delayed re-feed of the LXe into the detector. Suppression by three orders of magnitude adds an additional $225 \mathrm{~kg}$ to the total xenon budget when cycling 1000 standard liter per minute.

- The $2 \nu \beta \beta$ decay spectrum of ${ }^{136} \mathrm{Xe}$ has been simulated assuming the measured half-life of $T_{1 / 2}=(2.165 \pm$ $0.061) \times 10^{21}$ years [25]. For the analytic spectrum we use the non-relativistic Primakoff-Rosen approximation for the interaction between nuclei and electrons in the parametrization discussed in [26]. This approximation is conservative as it overestimates the rate around the spectral end point.

- ${ }^{222} \mathrm{Rn}$ in LXe is assumed to be reduced by online cryogenic distillation [27] and stringent material selection to a concentration equivalent to $0.1 \mu \mathrm{Bq}^{222} \mathrm{Rn}$ activity per $\mathrm{kg}$ of xenon. Being crucial for the WIMP search, significant efforts are being undertaken to reach this design goal. The dominant intrinsic background contribution for the $0 \nu \beta \beta$ search originates from the $\beta$-decay of ${ }^{214} \mathrm{Bi}\left(\mathrm{Q}_{\beta}=\right.$ $3.27 \mathrm{MeV}$ ). In $19.1 \%$ of the cases it decays to the ${ }^{214} \mathrm{Po}$ ground state without $\gamma$-emission, which renders the rejection based on spatial topology rather inefficient, as discussed in Sect. 3. The short half-life of the decay daughter ${ }^{214} \mathrm{Po}\left(T_{1 / 2}=164.3 \mu \mathrm{s}\right)$, however, allows for BiPo event tagging and suppression with more than $99.8 \%$ efficiency [28].

\subsection{External radiogenic background sources}

Long-lived radionuclides are present in each detector material. Their decays, as well as the subsequent decays of their daughter isotopes, might introduce background in the target. Activity levels for all materials are listed in Table 2 and based on reports from previous or ongoing experiments $[13,14]$.

- The natural decay chains of ${ }^{238} \mathrm{U},{ }^{232} \mathrm{Th}$ and ${ }^{235} \mathrm{U}$ yield a background contribution primarily from $\gamma$-rays emitted by ${ }^{214} \mathrm{Bi}-\left(\mathrm{E}_{\gamma}=2.45 \mathrm{MeV}\right)$ and ${ }^{208} \mathrm{Tl}$-decays $\left(\mathrm{E}_{\gamma}=\right.$ $2.61 \mathrm{MeV})$. The former two chains were split into their early and late component at ${ }^{226} \mathrm{Ra}$ and ${ }^{228} \mathrm{Th}$, respectively, to account for radiogenic non-equilibrium.

- ${ }^{60} \mathrm{Co} \beta$-decays dominantly $(99.95 \%)$ via the two excited states of ${ }^{60} \mathrm{Ni}$. The de-excitation is temporally nonresolvable and spatial coincidences of the $1.17 \mathrm{MeV}$ and the $1.33 \mathrm{MeV} \gamma$-events contribute to the background.

- Among the radio-isotopes from cosmogenic material activation at sea level [29], ${ }^{44} \mathrm{Ti}$ in the cryostat material is the most relevant, due to its long half-life $\left(T_{1 / 2}\right.$ $=59.1$ years) and the subsequent decay of ${ }^{44} \mathrm{Sc}$ with $\gamma$ emission at $2.66 \mathrm{MeV}$.

- ${ }^{222} \mathrm{Rn}$ contamination in the non-instrumented xenon surrounding the TPC can contribute to the ${ }^{214} \mathrm{Bi}$-induced $\gamma$-background. The rejection based on BiPo tagging described above cannot be applied since the subsequent alpha decays are not observed.

\section{Analysis and background results}

The background sources discussed in Sect. 4 are simulated with the GEANT4 particle physics simulation toolkit [30], using the detector model presented in Sect. 2.1. The equivalent of at least 100 years of DARWIN run time has been simulated for each material and isotope. In this section, we discuss the methods applied for event selection. The analytical background model, used for the profile-likelihood analy- 
sis in Sect. 6.2, is also described, and the background results are discussed.

\subsection{Monte Carlo data processing and event selection}

The energy depositions generated by GEANT4 per event undergo a density-based spatial clustering algorithm [19] to topologically distinguish signal-like single site (SS) from background-like multi site (MS) events, as discussed in Sect. 3. We assume a separation threshold $\epsilon=15 \mathrm{~mm}$ for the DARWIN TPC. This comparatively coarse clustering inevitably results in a fraction of $\gamma$-accompanied $\beta$-decays from background events, e.g., ${ }^{214} \mathrm{Bi}$ decays which frequently occur with higher multiplicity, being falsely identified as SS and consequentially contributing to the background.

To account for the finite energy resolution of the detector, the combined energy deposited inside each cluster is smeared according to a resolution of

$\frac{\sigma_{E}}{E}=\frac{a}{\sqrt{E[\mathrm{keV}]}}+b$,

with $a=(0.3171 \pm 0.0065)$ and $b=(0.0015 \pm 0.0002)$. At $E=Q_{\beta \beta}$ this corresponds to $\sigma_{E} / E=0.8 \%$, as demonstrated in the XENON1T TPC [31]. The cluster position is smeared to account for the detector's spatial resolution which is conservatively assumed to be $\sigma_{x, y}=\sigma_{z}=10 \mathrm{~mm}$ above $2 \mathrm{MeV}$.

Constraining the target to a super-ellipsoidal-shaped fiducial volume (FV) allows us to exploit the excellent selfshielding capabilities of liquid xenon. To compensate for the reduced shielding power in the xenon gas phase, the FV is shifted slightly downwards from the center of the instrumented volume. The fiducial volume is optimized for each FV mass independently. We use the lifetime-weighted combined external background, after the selection of single site events, energy and spatial resolution smearing. Only events with an energy inside the $0 \nu \beta \beta$-ROI of [2435-2481] keV, defined as the full width at half maximum (FWHM) range of the expected signal peak, are considered. The spatial distribution of external background events inside the active volume is shown in Fig. 4.

\subsection{Background model and fiducial mass dependence}

The selection of events within a fiducial volume removes all $\alpha$ - and $\beta$-contributions originating from external sources. The $\gamma$-background is shown in Fig. 5 (bottom) for the $20 \mathrm{t}$ fiducial volume. In the $0 v \beta \beta$-ROI the background is composed of the absorption peak from ${ }^{214} \mathrm{Bi}$ at $E_{\mathrm{Bi}}=2.45 \mathrm{MeV}$ and Compton scattered photons, mainly from the ${ }^{208} \mathrm{Tl}$ line $\left(E_{\mathrm{Tl}}=2.61 \mathrm{MeV}\right)$. Compton scatterings inside the fiducial volume with the subsequent escape of the scattered lower

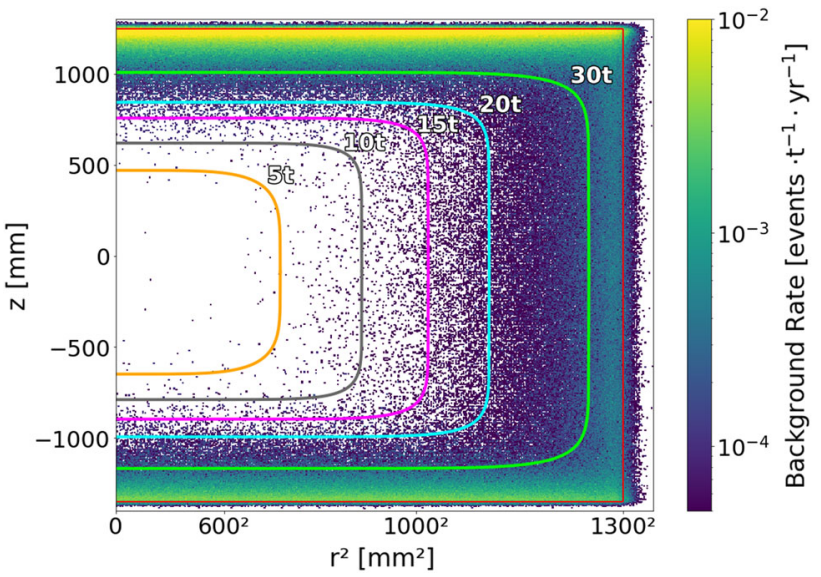

Fig. 4 Spatial distribution of external background events inside the instrumented volume for 100 years of DARWIN run time. The colored lines indicate the contours of the optimized fiducial volumes containing different LXe target masses. The $5 \mathrm{t}$ fiducial volume is used for the sensitivity estimate presented below

energy $\gamma$-ray are strongly suppressed by fiducialization. The continuous background is dominated by photons that undergo an undetected Compton scatter outside the detector followed by their absorption in the fiducial volume.

The continuous contribution from $\gamma$-rays emitted in ${ }^{44} \mathrm{Sc}$ decays accounts for less than $1 \%$ of the external background. ${ }^{214} \mathrm{Bi}$ decays with $E_{\gamma}>Q_{\beta \beta}$ contribute with a similarly subdominant level. Spatial coincident absorption of both ${ }^{60} \mathrm{Co}$ gammas accounts for only approximately $10^{-3}$ of the total material background at $E=2.51 \mathrm{MeV}$ in the $30 \mathrm{t}$ fiducial volume. In the fiducial volume mass range of interest, it can be considered negligible. The largest background contribution in the ROI is induced by the absorption peak of $2.45 \mathrm{MeV}$ $\gamma$-rays emitted by ${ }^{214} \mathrm{Bi}$ decays in the detector materials. The contribution from ${ }^{214} \mathrm{Bi}$ decays in the non-instrumented LXe around the TPC accounts for approximately $0.1 \%$ of the total material-induced background.

The relative contributions to the $\gamma$-background in the ROI are shown per material of origin in Fig. 5 (top). The similar contribution of cryostat-induced events from the walls and the combined PMT and electronics background originating from the top and bottom sensor array is a result of the optimization of the fiducial volume, which is properly balancing the $r$ - and $z$-extent.

The spectral shape of the material-induced $\gamma$-background is modelled with a Gaussian peak and an exponentially decreasing continuum for each line, as shown in Fig. 5 (bottom). We consider the $2.61 \mathrm{MeV}^{208} \mathrm{Tl}$ peak, the $2.66 \mathrm{MeV}$ ${ }^{44} \mathrm{Sc}$ peak and each contribution of ${ }^{214} \mathrm{Bi}$ with $E_{\gamma}>$ 2.0 MeV. The ratio between the ${ }^{214} \mathrm{Bi}$ and the ${ }^{44} \mathrm{Sc}$ peaks to the ${ }^{208} \mathrm{Tl}$ peak intensity is established using Monte Carlo data in fiducial volumes sufficiently large to provide high statistics. Similarly, each continuum contribution is tied to 

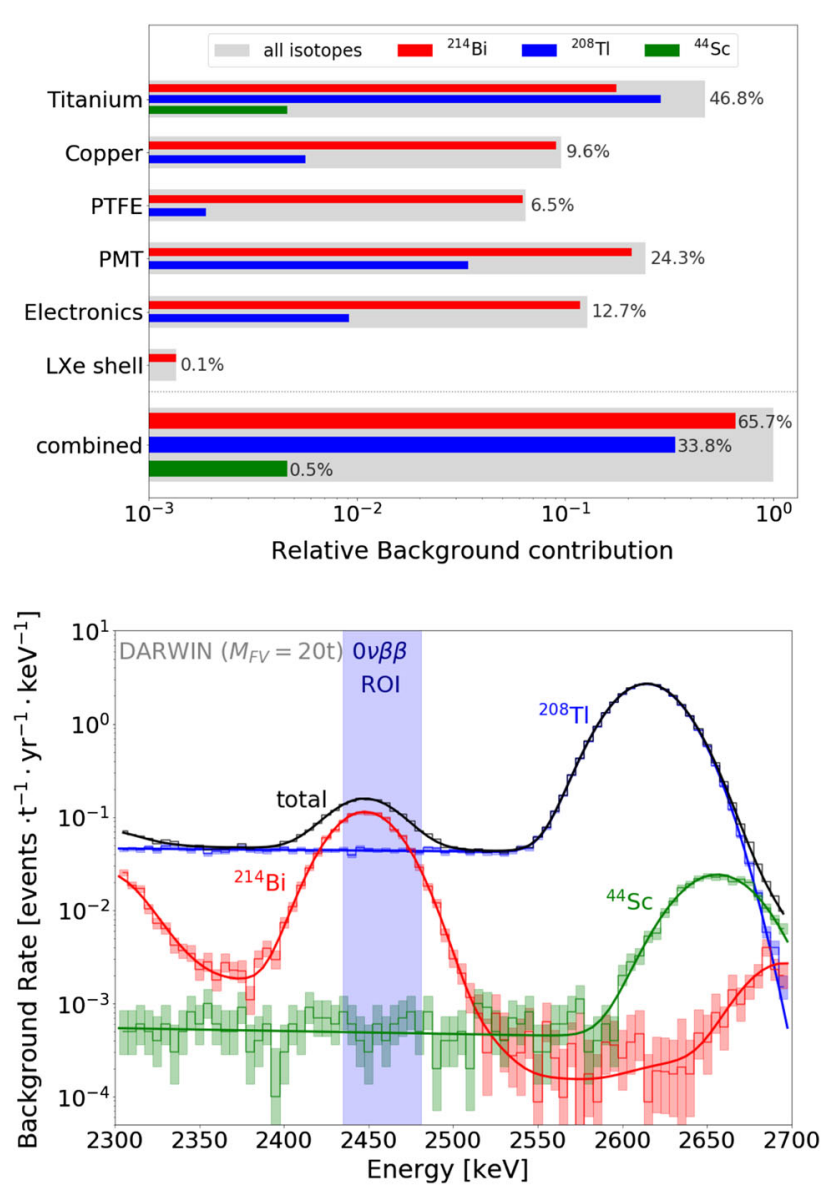

Fig. 5 Composition of the material-induced external background in the $20 \mathrm{t}$ fiducial volume. Top: Relative contribution to the background in the $0 \nu \beta \beta$-ROI by material and isotope. Bottom: Background spectra by isotope with the corresponding model fits. The relative contributions and spectral shapes are representative for smaller fiducial volumes

its corresponding peak intensity and a fixed relation between the three slope parameters is found. The only remaining free parameters of the combined model are the total intensity of the ${ }^{208} \mathrm{Tl}$ peak and one common slope parameter. The model is tested and confirmed using a $\chi^{2}$ goodness-of-fit test on the combined external background in the fiducial mass range $\leq 20 t$.

The intrinsic background from ${ }^{8} \mathrm{~B}$ neutrinos is assumed to be flat. The spectra corresponding to ${ }^{137} \mathrm{Xe}$ and ${ }^{222} \mathrm{Rn}$ are approximated linearly falling in the $[2.2-2.8 \mathrm{MeV}]$ range. The slopes are obtained from Monte Carlo studies. The $2 v \beta \beta$ spectrum is convolved with the Gaussian energy resolution.

The suppression of the external background with decreasing fiducial mass is shown in Fig. 6, together with the target mass independent intrinsic contributions.

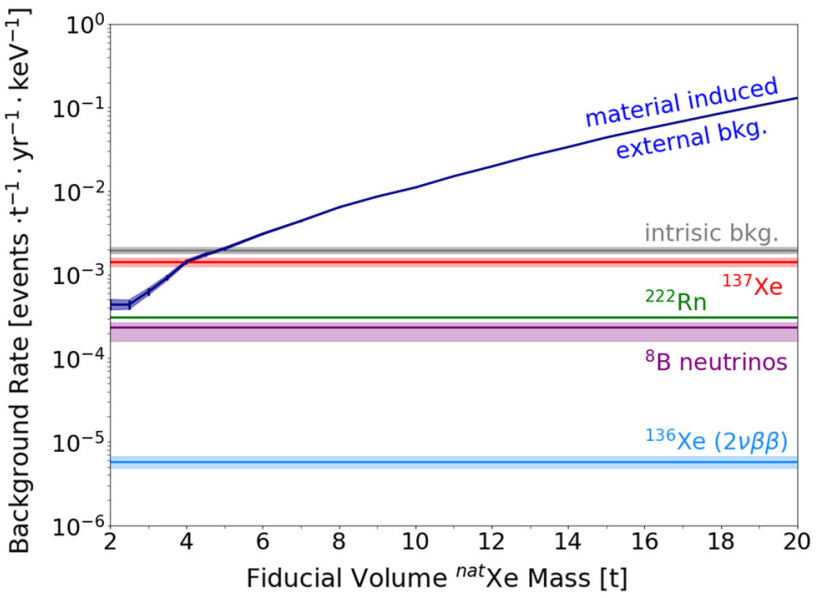

Fig. 6 Background rate in the ROI versus fiducial mass. External contributions are combined. Fiducial volume independent intrinsic sources are shown per contribution. Bands indicate $\pm 1 \sigma$ uncertainties. At $5 \mathrm{t}$, the external sources contribute at the same level as the combined intrinsic background

\subsection{Background rates in the $0 v \beta \beta$-ROI}

The fiducial volume is optimized for $T_{1 / 2}^{0 v}$ sensitivity, as discussed in detail in Sect. 6.1, and yields $5 \mathrm{t}$. The resulting background spectrum from intrinsic and external sources is shown for this fiducial mass in Fig. 7.

The intrinsic background in the ROI is dominated by the gently falling $\beta^{-}$-spectrum of ${ }^{137} \mathrm{Xe}$ decay. Subdominant contributions are the electron scattering of solar ${ }^{8} \mathrm{~B}$ neutrinos and $\beta^{-}$-events from ${ }^{214} \mathrm{Bi}$-decays which are not vetoed by BiPo tagging. The $2 \nu \beta \beta$ spectrum overlaps negligibly with the ROI, but dominates the background toward lower energies.

The model-estimated background indices for all contributions are summarized in Table 3. To validate the analytic model introduced in Sect. 5.2, we compare the background model estimate with the values derived by weighted event counting in the $5 \mathrm{t}$ fiducial mass data from Monte Carlo. Both results are in agreement within the statistical errors. The model-derived uncertainty on the background, however, is a factor of 4 lower than the Poissonian statistics error in the simple counting approach. The uncertainties on intrinsic background sources account for statistical errors, the variation of the overlap with the $0 v \beta \beta$-ROI based on the energy resolution and systematic uncertainties from (theory-driven) input parameters. The dominant contributions are the $v_{e}$ survival probability and the neutrino flux $\left({ }^{8} \mathrm{~B} v-e^{-}\right.$scattering $)$, the ${ }^{136} \mathrm{Xe}$ neutron capture cross-section (governing the ${ }^{137} \mathrm{Xe}$ production rate) and the half-life of ${ }^{136} \mathrm{Xe}(2 v \beta \beta$ decay). 
Table 3 Expected background index averaged in the $0 \nu \beta \beta$-ROI of [2435-2481] keV, corresponding event rate in the $5 \mathrm{t} \mathrm{FV}$ and relative uncertainty by origin

\begin{tabular}{|c|c|c|c|}
\hline Background source & $\begin{array}{l}\text { Background index } \\
\text { [events/(t.year.keV })]\end{array}$ & $\begin{array}{l}\text { Rate } \\
\text { [events/year] }\end{array}$ & Rel. uncertainty \\
\hline \multicolumn{4}{|l|}{ External sources $(5 t F V)$ : } \\
\hline${ }^{214} \mathrm{Bi}$ peaks + continuum & $1.36 \times 10^{-3}$ & 0.313 & $\pm 3.6 \%$ \\
\hline${ }^{208} \mathrm{Tl}$ continuum & $6.20 \times 10^{-4}$ & 0.143 & $\pm 4.9 \%$ \\
\hline${ }^{44} \mathrm{Sc}$ continuum & $4.64 \times 10^{-6}$ & 0.001 & $\pm 15.8 \%$ \\
\hline \multicolumn{4}{|l|}{ Intrinsic contributions: } \\
\hline${ }^{8} \mathrm{~B}(v-e$ scattering $)$ & $2.36 \times 10^{-4}$ & 0.054 & $+13.9 \%,-32.2 \%$ \\
\hline${ }^{137} \mathrm{Xe}$ ( $\mu$-induced $n$-capture $)$ & $1.42 \times 10^{-3}$ & 0.327 & $\pm 12.0 \%$ \\
\hline${ }^{136} \mathrm{Xe} 2 \nu \beta \beta$ & $5.78 \times 10^{-6}$ & 0.001 & $+17.0 \%,-15.2 \%$ \\
\hline${ }^{222} \mathrm{Rn}$ in $\mathrm{LXe}(0.1 \mu \mathrm{Bq} / \mathrm{kg})$ & $3.09 \times 10^{-4}$ & 0.071 & $\pm 1.6 \%$ \\
\hline Total & $3.96 \times 10^{-3}$ & 0.910 & $+4.7 \%,-5.0 \%$ \\
\hline
\end{tabular}

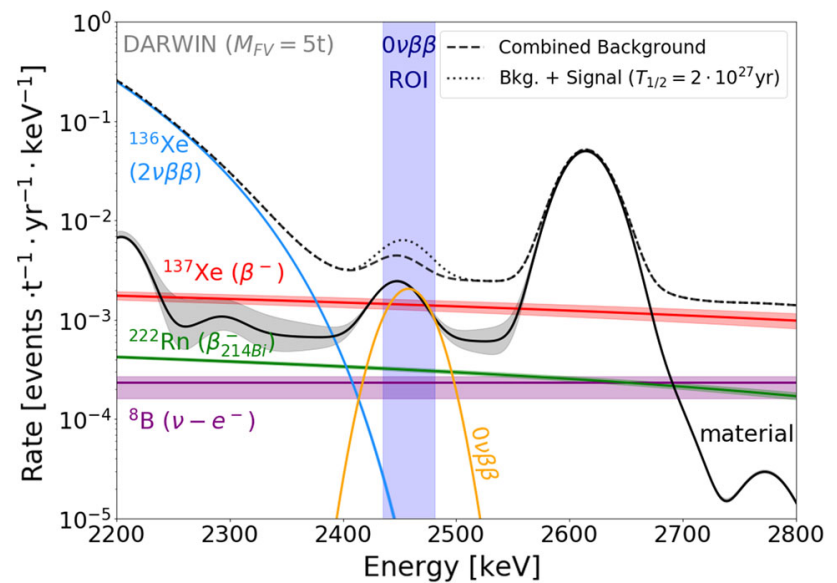

Fig. 7 Predicted background spectrum around the $0 \nu \beta \beta$-ROI for the $5 \mathrm{t}$ fiducial volume. A hypothetical signal of 0.5 counts per year corresponding to $T_{1 / 2}^{0 v} \approx 2 \times 10^{27}$ years is shown for comparison. Bands indicate $\pm 1 \sigma$ uncertainties

\section{Sensitivity calculation}

We use the background rates predicted in Sect. 5.3 to derive a limit on the half-life sensitivity at $90 \%$ confidence level (CL) as well as the $3 \sigma$ discovery potential for the $0 \nu \beta \beta$-decay. The latter is defined as the minimal value of $T_{1 / 2}^{0 v}$ required to exclude the null hypothesis with a median significance of $99.7 \%$ CL.

\subsection{Half-life sensitivity estimation}

Based on the figure-of-merit estimator proposed in [32] we calculate the half-life sensitivity at $90 \%$ CL as:

$T_{1 / 2}^{0 v}=\ln 2 \frac{\epsilon f_{\mathrm{ROI}} \alpha N_{A}}{1.64 M_{\mathrm{Xe}}} \frac{\sqrt{M t}}{\sqrt{B \Delta E}}$,

with $\epsilon=0.9$ being the detection efficiency of a single site $0 \nu \beta \beta$-decay event, $f_{\mathrm{ROI}}=0.76$ the fraction of signal cov- ered by the ROI, $\alpha=0.089$ the abundance of ${ }^{136} \mathrm{Xe}$ in natural xenon, $N_{A}$ the Avogadro number in $\mathrm{mol}^{-1}, M_{\mathrm{Xe}}$ the molar mass number of xenon in $\mathrm{t} / \mathrm{mol}, M$ the fiducial mass in tons, $t$ the exposure time in years, $B$ the background index in $\mathrm{t}^{-1}$ year $^{-1} \mathrm{keV}^{-1}$, and $\Delta E$ the width of the ROI in $\mathrm{keV}$. The value 1.64 is the number of standard deviations corresponding to a $90 \% \mathrm{CL}$.

Following Eq. (3) and using the background index for the $5 \mathrm{t}$ fiducial mass (Table 3 ), we obtain a half-life sensitivity of $2.0 \times 10^{27}$ years $\left(1.3 \times 10^{27}\right.$ years $)$ after $10(4)$ years of exposure.

This figure-of-merit estimation is an established tool to directly compare $0 \nu \beta \beta$ sensitivities of different experiments using common statistical methods and assumptions. It also allows for a straightforward assessment of the sensitivity as a function of different parameters, such as the fiducial mass. It does not, however, consider background uncertainties, but assumes perfect knowledge of the background rates.

\subsection{Frequentist profile-likelihood analysis}

To account for and effectively constrain the background uncertainties, we apply a profile-likelihood analysis based on the background model discussed in Sect. 5.2. The inserted signal is a Gaussian peak with $Q_{\beta \beta}$ and $\sigma_{E}\left(Q_{\beta \beta}\right)$ according to Eq. (2), which is scaled by the ${ }^{136} \mathrm{Xe}$ atoms in the target volume, an activity corresponding to $T_{1 / 2}^{0 v}$ and the detection efficiency, as shown in Fig. 7.

Background uncertainties from the model are treated as nuisance parameters with Gaussian constraining terms in the likelihood. For external background contributions, their variances are obtained either by the model fit on the spectrum corresponding to $5 \mathrm{t} \mathrm{FV}\left({ }^{208} \mathrm{Tl}\right.$ peak intensity and slope parameter) or extrapolation of the model parameters from larger fiducial volumes into the low fiducial mass range $\left({ }^{214} \mathrm{Bi} /\right.$ ${ }^{208} \mathrm{Tl}$ peak ratio, ${ }^{208} \mathrm{Tl}$ continuum $/{ }^{208} \mathrm{Tl}$ peak intensity). The uncertainty on the subdominant contribution from ${ }^{44} \mathrm{Sc}$ has 
been neglected. For the intrinsic contributions, the variances correspond to the square of the errors listed in Table 3. The corresponding slope uncertainties are negligible.

We obtain a $T_{1 / 2}^{0 v}$ sensitivity limit of $2.4 \times 10^{27}$ years for a 10 years exposure with $5 \mathrm{t}$ fiducial mass. The corresponding $3 \sigma$ discovery potential after 10 years exposure is $1.1 \times 10^{27}$ years.

\section{Discussion}

The DARWIN observatory will reach a sensitivity to the neutrinoless double beta decay of ${ }^{136} \mathrm{Xe}$ of $2.4 \times 10^{27}$ years $T_{1 / 2}$ exclusion limit (90\% CL) and a discovery sensitivity $(3 \sigma)$ of $T_{1 / 2}=1.1 \times 10^{27}$ years after 10 years of exposure.

In the baseline scenario discussed above, the assumptions on radio-purity and detector performance are considered realistic or even conservative. In an optimistic scenario, the external background could be reduced by a factor of three or more. The required measures include the use of less radioactive PMTs (with reduced mass of ceramic [33]) and/or low radioactivity SiPMs, more stringent material selection to reach lower levels of radio-activity for PTFE [34], copper [35] and titanium, as well as more radio-pure electronics.

Intrinsic backgrounds, dominated by the muon-induced activation of ${ }^{136} \mathrm{Xe}$, are difficult to mitigate assuming the muon flux at 3500 meter water equivalent (mwe) depth of LNGS. A time- and spatial- muon veto might allow for suppression by up to a factor of two at an acceptable exposure loss. The ${ }^{137} \mathrm{Xe}$ contribution would, however, become subdominant in a sufficiently deep laboratory. A total intrinsic background suppression by a factor of five or even eight could then be reached assuming a reduced BiPo tagging inefficiency of $0.1 \%$ and $0.01 \%$, respectively. Assuming a factor five reduction in external sources the latter scenario leads to a solar ${ }^{8} \mathrm{~B}$ neutrino dominated background.

The sensitivity could be increased by further exploitation of the SS/MS discrimination, discussed in Sect. 3. Despite increased signal rejection, the gain in background reduction dominates for spatial separation thresholds down to $\epsilon=3 \mathrm{~mm}$. The cluster separation in the $x$-y-plane would benefit from a higher granularity photosensor top array, featuring e.g. SiPMs. The $z$-position reconstruction is already more accurate and a combined three dimensional charge signal analysis will optimize the separation.

The largest sensitivity increase can be achieved with a combination of the above mentioned measures. Figure 8 shows the fiducial volume mass dependency (top) and time evolution (bottom) of the DARWIN half-life limit sensitivity ( $90 \%$ CL) calculated with the figure-of-merit estimator (see Sect. 6.1) for the baseline and different optimistic scenarios. The latter assume reduced spatial separation threshold $\epsilon$, intrinsic and external background rates. Figure 9 trans-
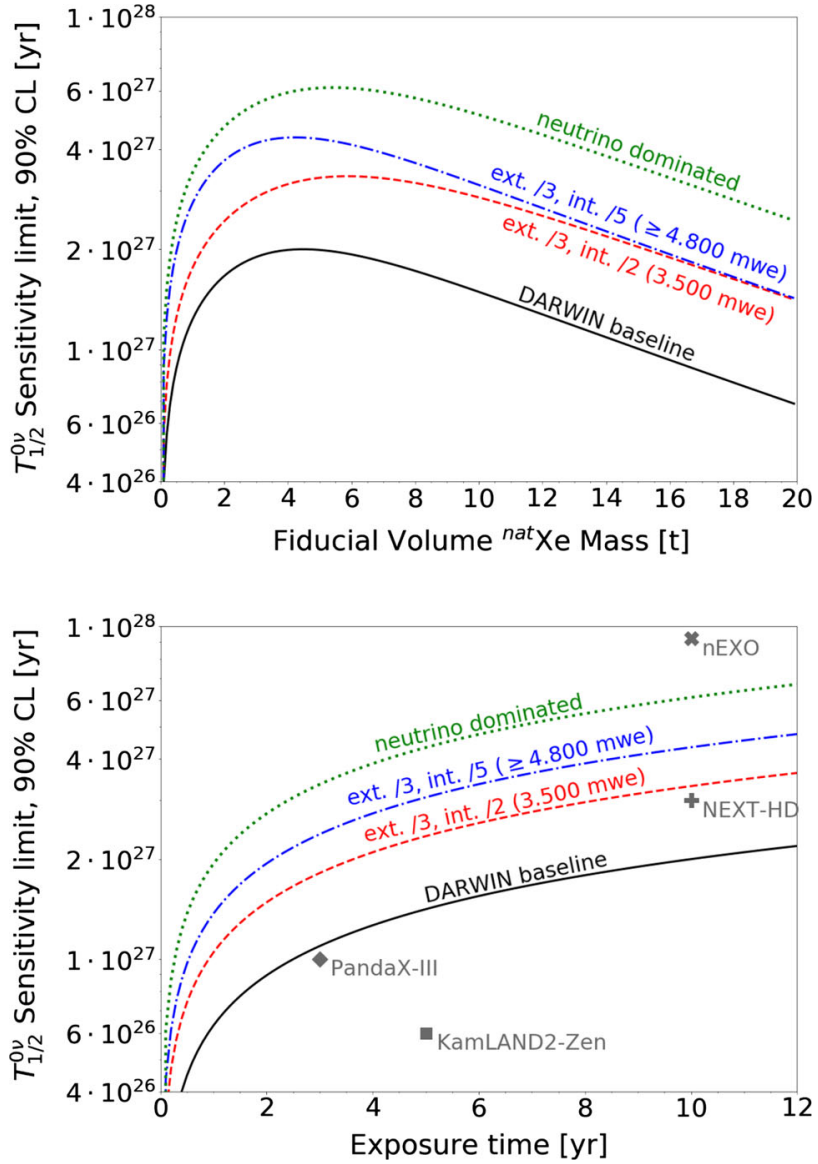

Fig. 8 DARWIN median $T_{1 / 2}^{0 v}$ sensitivity at $90 \% \mathrm{CL}$ as a function of fiducial volume mass for 10 years of exposure (top) as well as of the exposure time for the optimized fiducial volume (bottom). The baseline design is compared with different optimistic scenarios. The latter assume a reduction of the external (ext.) and the intrinsic (int.) backgrounds and improved spatial separation threshold of $10 \mathrm{~mm}$ (red, blue) or $5 \mathrm{~mm}$ (green). Sensitivity projections for future ${ }^{136} \mathrm{Xe} 0 \nu \beta \beta$ experiments are shown for comparison [8-10,37]

lates the half-life limit sensitivity to the effective Majorana neutrino mass $m_{\beta \beta}$ using Eq. (1), where the $m_{\beta \beta}$ range corresponds to the range of published nuclear matrix elements [36]. Under the conservative baseline assumptions, DARWIN reaches a $m_{\beta \beta}$ limit of [18-46 meV]. The neutrino dominated scenario yields a limit in the [11-28 meV] range. Future dedicated neutrinoless double beta decay experiments using either ${ }^{136} \mathrm{Xe}$ or other isotopes are aiming for a similar science reach as DARWIN, as shown for comparison in Table 4 and in Fig. 8 (bottom).

The objective of detecting particle dark matter with a sensitivity down to the neutrino floor requires the DARWIN observatory to be an ultra-low background experiment. It additionally features a high ${ }^{136} \mathrm{Xe}$ target mass, excellent energy resolution and single site discrimination capability. In the presented baseline scenario DARWIN will reach a sensitivity that approaches that of the tonne-scale proposed $0 \nu \beta \beta$ 
Table 4 Comparison of $T_{1 / 2}^{0 \nu}$ and $m_{\beta \beta}$ sensitivity limits (90\% CL) between DARWIN and future $0 v \beta \beta$ experiments. For experiments using ${ }^{136}$ Xe the $m_{\beta \beta}$ ranges are calculated with the nuclear matrix element ranges from [36], those using other isotopes are taken from [37]

\begin{tabular}{|c|c|c|c|c|c|}
\hline \multirow[t]{2}{*}{ Experiment } & \multirow[t]{2}{*}{ Isotope } & \multicolumn{2}{|c|}{ Sensitivity limit (90\% CL) } & \multirow{2}{*}{$\begin{array}{l}\text { Exposure time } \\
\text { [year] }\end{array}$} & \multirow[t]{2}{*}{ Reference } \\
\hline & & $T_{1 / 2}^{0 v}[$ year] & $m_{\beta \beta}[\mathrm{meV}]$ & & \\
\hline DARWIN (baseline) & ${ }^{136} \mathrm{Xe}$ & $2.4 \times 10^{27}$ & $18-46$ & 10 & this work \\
\hline DARWIN ( $v$ dominated) & ${ }^{136} \mathrm{Xe}$ & $6.2 \times 10^{27}$ & $11-28$ & 10 & this work \\
\hline KamLAND2-Zen & ${ }^{136} \mathrm{Xe}$ & $6 \times 10^{26}$ & $37-91$ & 5 & [37] \\
\hline PandaX-III & ${ }^{136} \mathrm{Xe}$ & $1 \times 10^{27}$ & $28-71$ & 3 & [9] \\
\hline NEXT-HD & ${ }^{136} \mathrm{Xe}$ & $3 \times 10^{27}$ & $16-41$ & 10 & [8] \\
\hline nEXO & ${ }^{136} \mathrm{Xe}$ & $9.2 \times 10^{27}$ & $9-23$ & 10 & {$[10]$} \\
\hline SNO+-II & ${ }^{130} \mathrm{Te}$ & $7 \times 10^{26}$ & $20-70$ & 5 & [37] \\
\hline AMoRE-II & ${ }^{100} \mathrm{Mo}$ & $5 \times 10^{26}$ & $15-30$ & 5 & {$[37]$} \\
\hline CUPID & ${ }^{130} \mathrm{Te} /{ }^{100} \mathrm{Mo}$ & $(2-5) \times 10^{27}$ & $6-17$ & 10 & [37] \\
\hline LEGEND-1000 & ${ }^{76} \mathrm{Ge}$ & $1 \times 10^{28}$ & $11-28$ & 10 & [37] \\
\hline
\end{tabular}

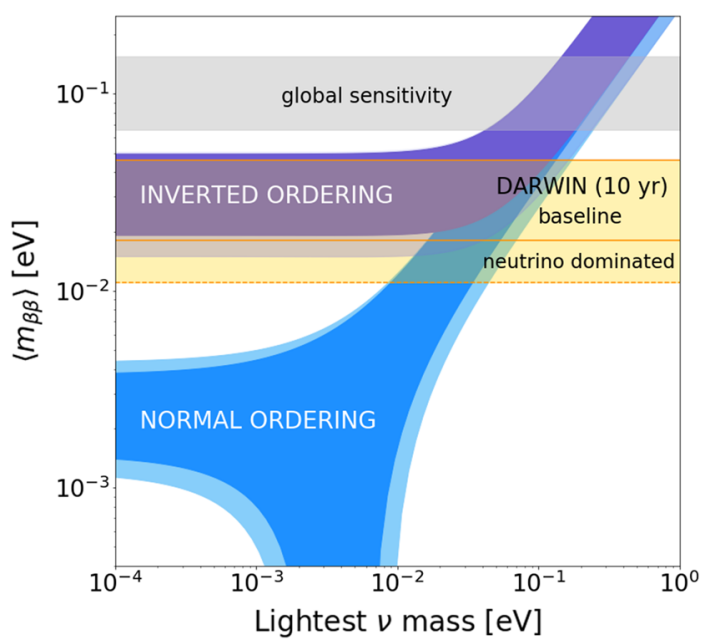

Fig. 9 Effective Majorana neutrino mass vs. lightest neutrino mass. The sensitivity reach after $50 \mathrm{t} \times$ years of exposure is shown for the baseline and the optimistic neutrino dominated scenario. The horizontal bands stem from the range of nuclear matrix elements [36]. Global sensitivity according to [38], oscillation parameters from [39,40]

experiments. Under more optimistic assumptions, requiring adaptations to the baseline design, DARWIN will explore the full inverted hierarchy and will compete with the most ambitious proposed $0 \nu \beta \beta$ projects.

Acknowledgements This work was supported by the Swiss National Science Foundation under Grants No 200020-162501 and No 200020175863, by the European Union's Horizon 2020 research and innovation programme under the Marie Sklodowska-Curie grant agreements No 674896, No 690575 and No 691164, by the European Research Council (ERC) grant agreements No 742789 (XENOSCOPE) and No 724320 (ULTIMATE), by the Max-Planck-Gesellschaft, by the Deutsche Forschungsgemeinschaft (DFG) under GRK-2149, by the US National Science Foundation (NSF) grants No 1719271 and No 1940209, by the Portuguese FCT, by the Netherlands Organisation for Scientific Research (NWO), by the Ministry of Education, Science and Technological Development of the Republic of Serbia and by grant ST/N000838/1 from Science and Technology Facilities Council (UK).

Data Availability Statement This manuscript has associated data in a data repository. [Authors' comment: Data is made available on reasonable request to the collaboration or the corresponding authors.]

Open Access This article is licensed under a Creative Commons Attribution 4.0 International License, which permits use, sharing, adaptation, distribution and reproduction in any medium or format, as long as you give appropriate credit to the original author(s) and the source, provide a link to the Creative Commons licence, and indicate if changes were made. The images or other third party material in this article are included in the article's Creative Commons licence, unless indicated otherwise in a credit line to the material. If material is not included in the article's Creative Commons licence and your intended use is not permitted by statutory regulation or exceeds the permitted use, you will need to obtain permission directly from the copyright holder. To view a copy of this licence, visit http://creativecomm ons.org/licenses/by/4.0/.

Funded by SCOAP ${ }^{3}$.

\section{References}

1. A .S. Barabash, Average and recommended half-life values for two neutrino double beta decay. Nucl. Phys. A 935, 52-64 (2015). https://doi.org/10.1016/j.nuclphysa.2015.01.001. arxiv: 1501.05133

2. R. Henning, Current status of neutrinoless double-beta decay searches. Rev. Phys. 1, 29-35 (2016). https://doi.org/10.1016/j. revip.2016.03.001

3. M.J. Dolinski, A.W.P. Poon, W. Rodejohann, Neutrinoless doublebeta decay: status and prospects. Annu. Rev. Nucl. (2019). https:// doi.org/10.1146/annurev-nucl-101918-023407. arxiv:1902.04097

4. M. Redshaw, E. Wingfield, J. McDaniel, E.G. Myers, Mass and double-beta-decay Q value of Xe-136. Phys. Rev. Lett. 98, 053003 (2007). https://doi.org/10.1103/PhysRevLett.98.053003

5. EXO-200 collaboration, G. Anton, et al., Search for Neutrinoless Double- $\beta$ Decay with the Complete EXO-200 Dataset. Phys. Rev. Lett. 123, 161802 (2019). https://doi.org/10.1103/PhysRevLett.98. 053003, arxiv: 1906.02723 
6. KAMLAND- ZEN collaboration, A. Gando et al., Search for majorana neutrinos near the inverted mass hierarchy region with KamLAND-Zen, Phys. Rev. Lett. 117 (2016) 082503, https:// doi.org/10.1103/PhysRevLett.117.109903,10.1103/PhysRevLett. 117.082503, arxiv: 1605.02889

7. NEXT collaboration, V. Alvarez et al., NEXT-100 Technical Design Report (TDR): Executive Summary, JINST 7, T06001 (2012) https://doi.org/10.1088/1748-0221/7/06/T06001, arxiv: 1202.0721

8. J. J. Gomez-Cadena, Status and prospects of the NEXT experiment for neutrinoless double beta decay searches, ArXiv (2019), arxiv: 1906.01743

9. X. Chen et al., PandaX-III: Searching for neutrinoless double beta decay with high pressure ${ }^{136}$ Xe gas time projection chambers. Sci. Chin. Phys. Mech. Astron. 60, 061011 (2017). arxiv:1610.08883

10. nEXO collaboration, J. B. Albert et al., Sensitivity and discovery potential of nEXO to neutrinoless double beta decay. Phys. Rev. C 97, 065503 (2018), https://doi.org/10.1103/PhysRevC.97.065503, arxiv: 1710.05075

11. DARWIN collaboration, J. Aalbers et al., DARWIN: towards the ultimate dark matter detector, JCAP 1611, 017 (2016), https://doi. org/10.1088/1475-7516/2016/11/017,[arxiv:1606.07001

12. M. Schumann, Dual-phase liquid xenon detectors for dark matter searches. JINST 9, C08004 (2014). https://doi.org/10.1088/ 1748-0221/9/08/C08004. arxiv: 1405.7600

13. LZ collaboration, D. S. Akerib et al., Identification of radiopure titanium for the LZ dark matter experiment and future rare event searches, Astropart. Phys. 96, 1-10 (2017), https://doi.org/ 10.1016/j.astropartphys.2017.09.002, arxiv:1702.02646

14. XENON collaboration, E. Aprile et al., Material radioassay and selection for the XENON1T dark matter experiment. Eur. Phys. J. C 77, 890 (2017), https://doi.org/10.1140/epjc/s10052-017-5329-0, arxiv: 1705.01828

15. L. Baudis, M. Galloway, A. Kish, C. Marentini, J. Wulf, Characterisation of silicon photomultipliers for liquid xenon detectors. JINST 13, P10022 (2018). https://doi.org/10.1088/1748-0221/13/ 10/P10022. arxiv: 1808.06827

16. L. Baudis et al., Performance of the Hamamatsu R11410 photomultiplier tube in cryogenic xenon environments. JINST 8, P04026 (2013). https://doi.org/10.1088/1748-0221/8/04/P04026. arxiv: 1303.0226

17. F. Arneodo, M. Benabderrahmane, G. Bruno, V. Conicella, A.D. Giovanni, O. Fawwaz et al., Cryogenic readout for multiple VUV4 Multi-Pixel Photon Counters in liquid xenon. Nucl. Instrum. Methods A 893, 117-123 (2018). https://doi.org/10.1016/j.nima.2018. 03.022. arxiv: 1707.08004

18. D. Ferenc, A. Chang and M. Šegedin Ferenc, The novel ABALONE photosensor technology: 4-year long tests of vacuum integrity, internal pumping and afterpulsing. ArXiv (2017) arxiv: 1703.04546

19. M. Ester, H.-P. Kriegel, J. Sander, X. Xu, A density-based algorithm for discovering clusters in large spatial databases with noise. In: KDD Proceedings, pp. 226-231, AAAI Press, (1996)

20. R. Arnold et al., Probing new physics models of neutrinoless double beta decay with supernemo. Eur. Phys. J. C 70, 927943 (2010). https://doi.org/10.1140/epjc/s10052-010-1481-5. arxiv: 1005.1241

21. Particle Data Group collaboration, M. Tanabashi et al., Review of particle physics. Phys. Rev. D 98, 030001 (2018), https://doi.org/ 10.1103/PhysRevD.98.030001

22. B. Dutta, L.E. Strigari, Neutrino physics with dark matter detectors. Annu. Rev. Nucl. (2019). https://doi.org/10.1146/ annurev-nucl-101918-023450. arxiv:1901.0

23. EXO- 200 collaboration, J. Albert et al., Cosmogenic Backgrounds to $0 v \beta \beta$ in EXO-200. JCAP 04, 029 (2016), https://doi.org/10. 1088/1475-7516/2016/04/029, arxiv:1512.06835]
24. XENON1T collaboration, M. Selvi, Study of the performances of the shield and muon veto of the XENON1T experiment. PoS IDM2010 053 (2011), https://doi.org/10.22323/1.110.0053

25. EXO-200 collaboration, J. B. Albert et al., Improved measurement of the $2 \nu \beta \beta$ half-life of ${ }^{136} \mathrm{Xe}$ with the EXO-200 detector. Phys. Rev. C 89015502 (2014), https://doi.org/10.1103/PhysRevC.89. 015502, arxiv: 1306.6106

26. F. Boehm, P. Vogel, Physics of Massive Neutrinos, 2nd edn. (Cambridge University Press, Cambridge, 1992)

27. XENON collaboration, E. Aprile et al., Online ${ }^{222} \mathrm{Rn}$ removal by cryogenic distillation in the XENON100 experiment. Eur. Phys. J. C C77 358 (2017), https://doi.org/10.1140/epjc/ s10052-017-4902-x, arxiv:1702.06942

28. L. Baudis et al., Neutrino physics with multi-ton scale liquid xenon detectors. JCAP 01, 044 (2014). https://doi.org/10.1088/ 1475-7516/2014/01/044. arxiv:1309.7024

29. C. Zhang, D. M. Mei, V. A. Kudryavtsev and S. Fiorucci, Cosmogenic activation of materials used in rare event search experiments. Astropart. Phys. 84 62-69 (2016), https://doi.org/10.1016/ j.astropartphys.2016.08.008, arxiv:1603.00098

30. GEANT4 collaboration, S. Agostinelli et al., GEANT4: a simulation toolkit. Nucl. Instrum. Methods A 506 250-303 (2003), https:// doi.org/10.1016/S0168-9002(03)01368-8

31. XENON collaboration, E. Aprile et al., Energy resolution and linearity in the $\mathrm{keV}$ to $\mathrm{MeV}$ range measured in XENON1T. ArXiv (2020), arxiv:2003.03825

32. F.T. Avignone, G.S. King, YuG Zdesenko, Next generation doublebeta decay experiments: metrics for their evaluation. New J. Phys. 7, 6 (2005). https://doi.org/10.1088/1367-2630/7/1/006

33. XENON collaboration, E. Aprile et al., Lowering the radioactivity of the photomultiplier tubes for the XENON1T dark matter experiment. Eur. Phys. J. C 75546 (2015), https://doi.org/10.1140/epjc/ s10052-015-3657-5, arxiv:1503.07698

34. M. Auger et al., The EXO-200 detector, part I: Detector design and construction. JINST 7, P05010 (2012). https://doi.org/10.1088/ 1748-0221/7/05/P05010. arxiv:1202.2192

35. Majorana collaboration, N. Abgrall et al., The Majorana Demonstrator radioassay program. Nucl. Instrum. Methods A 828 22-36 (2016), https://doi.org/10.1016/j.nima.2016.04.070, arxiv: 1601.03779

36. J. Engel and J. Menéndez, Status and future of nuclear matrix elements for neutrinoless double-beta decay: a review. Rept. Prog. Phys. 80046301 (2017), https://doi.org/10.1088/1361-6633/ aa5bc5, arxiv: 1610.06548

37. A.S. Barabash, Possibilities of future double beta decay experiments to investigate inverted and normal ordering region of neutrino mass. Front. Phys. 6, 160 (2019). https://doi.org/10.3389/ fphy.2018.00160. arxiv:1901.11342

38. M. Agostini et al., Probing Majorana neutrinos with double- $\beta$ decay. Science 365, 1445-1448 (2019). https://doi.org/10.1126/ science.aav8613

39. I. Esteban, M. C. Gonzalez-Garcia, A. Hernandez-Cabezudo, M. Maltoni and T. Schwetz, Global analysis of three-flavour neutrino oscillations: synergies and tensions in the determination of $\theta_{23}, \delta_{C P}$, and the mass ordering. JHEP 01106 (2019), https://doi. org/10.1007/JHEP01(2019)106, arxiv: 1811.05487

40. NuFIT 4.1 (2019), www.nu-fit.org 